\title{
Anti-windup synthesis for systems with rate-limits using Riccati equations
}

\author{
Jorge Sofrony ${ }^{\mathrm{b}}$ Matthew C. Turner ${ }^{\mathrm{a}}$ Ian Postlethwaite ${ }^{\mathrm{a}}$ \\ ${ }^{a}$ Department of Engineering, University of Leicester, Leicester, LE1 7RH, UK. \\ ${ }^{\mathrm{b}}$ Universidad Nacional de Colombia, Sede Bogota, Av Carrera 30 No. 45-03, Colombia
}

\begin{abstract}
This paper develops an anti-windup scheme for systems with rate-limited actuators. The main results show how a full-order anti-windup compensator can be synthesised using an algebraic Riccati equation and several free parameters. A further result then shows how the free parameters may be chosen to influence, in an intuitive way, the local $\mathcal{L}_{2}$ gain of the system and the size of the region of attraction. The results are of interest due to their low computational burden and hence their applicability to systems of large state dimension.
\end{abstract}

Key words: Anti-windup compensation; Rate-saturation; Flight control.

\section{Introduction}

Much of the constrained input literature addresses the problem of input saturation whereby the magnitude of the control vector is subjected to element-wise constraints. Not surprisingly, researchers have devoted much attention to this problem due to its prevalence in most engineering applications. The magnitude saturation problem led practitioners and researchers to develop compensators which could be retrofitted [10] to existing controllers to assist the original controller in maintaining stability and performance during periods of saturation. These compensators have become known as "anti-windup" (AW) compensators and many synthesis techniques have been proposed (some of the notable examples are $[18,42,14,20,9,30,7,21,17]$ ). It is evident that at present, at least in principle, the control engineer who faces magnitude saturation problems has tools to enable him/her to address the problem, with some of the more modern AW schemes guaranteeing stability and performance.

Magnitude limits on the control vector are not the only class of input nonlinearity which may be present in an otherwise linear system. Another significant class of nonlinearity which is well known to cause problems is input rate saturation. Although there are various ways of modeling this phenomenon, in essence, rate saturation (or rate-limiting as it is often called) limits, normally in an element-wise fashion, the rate of change of the control vector. This 
type of nonlinearity is not as wide-spread as the saturation nonlinearity, as, for example, electrical systems typically are less prone to suffer from this problem. Nevertheless, rate-limited actuators can be a significant problem in many systems, particularly those with mechanical actuators, and in some applications rate-limiting can have devastating consequences. Some of the more notorious examples of the catastrophic effects of rate-limiting are the crashes of the SAAB Grippen [1] and Boeing V22 Osprey aircraft [3], and the meltdown of the Chernobyl nuclear power plant [35].

Recently, some attempts at applying anti-windup (AW) schemes to the rate saturation problem have been made $([2,16,37,38])$. Although the development of these schemes can be regarded as a step towards bridging the gap between the need to provide rigorous guarantees of the closed-loop system's behaviour during rate-saturation, and the need to develop techniques which are useful in practice, these schemes have tended to be lacking in intuition and have not been developed enough for the practising engineer. One of main advantages of using AW conditioning schemes is that the linear performance (i.e. the performance when rate-saturation does not occur) is not directly restricted by the conditioning method, giving full freedom in the design of the linear controller. Moreover, the AW compensation schemes advocated in [44] which we follow here, are designed independently of the linear controller and thus the same AW compensator can be used with multiple controllers. This can be useful in some practical systems where the linear controller may change, depending on operating condition.

The anti-windup technique developed for systems with rate-limits in this paper is based on the framework introduced in [44] and the Riccati equation synthesis method proposed in [33]. In order to make the method of [33] applicable to rate-limited systems we use fairly standard results on sector narrowing - see $[22,15,45]$ for example - where the deadzone nonlinearity is described locally by a sector of reduced size, leading to a tighter bound. By combining these various results, an AW design framework which is both rigorous and intuitive is developed. In particular, the AW technique proposed contains several tuning variables which can be adjusted in a reasonably straightforward manner in order to trade-off features important in practical anti-windup problems. In fact, the results in this paper were developed with the aim of implementing anti-windup compensators on a sophisticated experimental aircraft; these results are reported elsewhere [34,5,6].

The paper is organised as follows. In the next section a discussion of linear systems subject to rate-limited actuators will be given. Following this, the anti-windup problem for rate-limited actuators will be introduced and a method for solving this based on the Riccati-based technique of [33] will be proposed. A simulation example then follows and the paper ends with some concluding remarks.

\subsection{Notation}

Notation is mainly standard throughout the paper. The notation $H(s) \in \mathcal{R} \mathcal{H}_{\infty}$ indicates that the transfer function $H(s)$ is real, rational and is analytic in the right-half-complex plane. We 
make extensive use of the saturation function defined as

$$
\operatorname{sat}_{\bar{u}}(u):=\left[\begin{array}{c}
\operatorname{sat}_{1, \bar{u}_{1}}\left(u_{1}\right) \\
\vdots \\
\operatorname{sat}_{m, \bar{u}_{m}}\left(u_{m}\right)
\end{array}\right] \text { and } \operatorname{sat}_{i, \bar{u}_{i}}\left(u_{i}\right)=\operatorname{sign}\left(u_{i}\right) \min \left(\left|u_{i}\right|, \bar{u}_{i}\right)
$$

The deadzone function is also frequently used and is defined as

$$
\operatorname{Dz}(u):=u-\operatorname{sat}(u)
$$

The paper considers mainly local anti-windup compensation which requires consideration of a region of attraction. For a nonlinear system $\dot{x}=f(x)$ with equilibrium point $x=0$, the region of attraction is defined as

$$
\mathcal{A}=\left\{x \in \mathbb{R}^{n}: \lim _{t \rightarrow \infty} x(t)=0\right\}
$$

The $\mathcal{L}_{2}$ norm of a vector $x(t)$ is defined as

$$
\|x\|_{2}:=\int_{0}^{\infty} x(t)^{\prime} x(t) d t
$$

and the induced $\mathcal{L}_{2}$ norm (or $\mathcal{L}_{2}$ gain) of a nonlinear operator, $\mathcal{H}$ as

$$
\|\mathcal{H}\|_{i, 2}:=\sup _{0 \neq x \in \mathcal{L}_{2}} \frac{\|H x\|_{2}}{\|x\|_{2}}
$$

Later in the paper, it is convenient to work with a local $\mathcal{L}_{2}$ gain which is defined as

$$
\|\mathcal{H}\|_{i, 2, \mathcal{W}}:=\sup _{0 \neq x \in \mathcal{L}_{2} \cap \mathcal{W}} \frac{\|H x\|_{2}}{\|x\|_{2}}
$$

where $\mathcal{W}$ is some subset of the input space of $\mathcal{H}$. It is clear that $\|\mathcal{H}\|_{i, 2, \mathcal{W}} \leq\|\mathcal{H}\|_{i, 2}$.

\section{Linear systems with rate-limited actuators}

\subsection{System modeling}

The paper is concerned with systems which take the form shown in Figure 1 which depicts a closed-loop system which is linear except for the rate-limit nonlinearity. The stabilisable, 


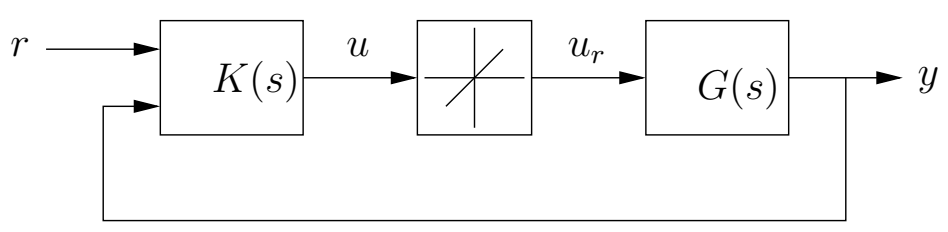

Fig. 1. Linear system with rate-limited actuators

detectable finite-dimensional linear time invariant (FDLTI) plant has state-space realisation

$$
G(s) \sim\left\{\begin{array}{l}
\dot{x}=A x+B u_{r} \\
y=C x+D u_{r}
\end{array}\right.
$$

where $x \in \mathbb{R}^{n_{p}}$ is the plant's state vector, $y \in \mathbb{R}^{q}$ is the vector of measured outputs and $u_{r} \in \mathbb{R}^{m}$ is the input to the plant. The controller $K(s)$ is also FDLTI and produces a control signal $u \in \mathbb{R}^{m}$ from a reference input $r \in \mathbb{R}^{n_{r}}$ and the measured output $y$. Disturbances have not been included, although are handled with little extra effort (see [44]). The input to the plant and the controller output are not necessarily identical due to the rate-limit nonlinearity, $\Phi($.$) :$ $\mathbb{R}^{m} \mapsto \mathbb{R}^{m}$, present at the plant input. This rate-limit nonlinearity represents the restrictions on the rates of the actuator and is well known to complicate stability and performance analysis of a system.

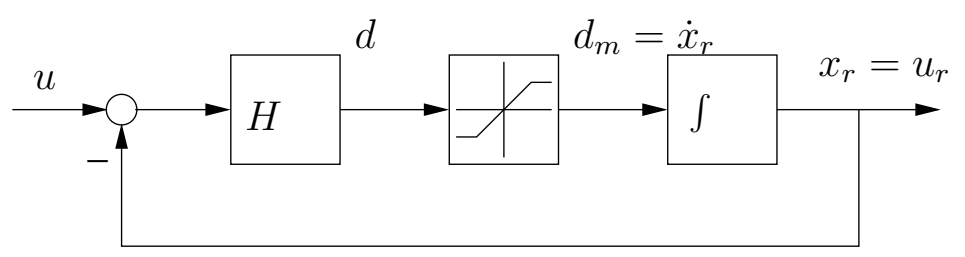

Fig. 2. Rate-limit model

Unlike the position saturation nonlinearity, rate saturation is a dynamic nonlinearity and several methods have been proposed to model it ([29,27,16,38]). Following [27] and others, in the work here it is useful to model the rate-limit nonlinearity as shown in Figure 2. This model of the rate-limiter features a (decentralised) magnitude saturation in a feedback loop with a set of integrators and a (decentralised) set of gains $H=\operatorname{diag}\left(h_{1}, \ldots, h_{m}\right)$. The rate-constraint values, which are now interpreted as magnitude saturation constraints in Figure 2, are defined as $\bar{d}_{i}, i \in\{1,2, \ldots, m\}$. The system's dynamic equations are as follows

$$
\Phi_{r}(u) \sim\left\{\begin{array}{l}
\dot{x}_{r}=\operatorname{sat}\left(-H x_{r}+H u\right) \\
u_{r}=x_{r}
\end{array}\right.
$$

This model of a rate-limit is appealing because, when the rate-limits have not been reached (i.e. when the saturation block in Figure 2 acts as the identity) the model behaves as a bank of low pass filters with cut-off frequency $1 / h_{i}$ in each channel. However, when the rate-limits have been reached, the rate-limit model can be interpreted as a restricted tracking problem: that is the output $u_{r}$ attempts to follow $u$ but with a restricted rate. 
Remark 1: The time constants $\frac{1}{h_{i}}$ must typically be greater than the dominant frequency components of $u$ in order to prevent the linear dynamics of the actuator from interfering too much with the nominal control system design. This is often a valid practical assumption but if not, some account of these linear dynamics must be taken during nominal linear controller design.

\subsection{Anti-windup architecture and the equivalent system}

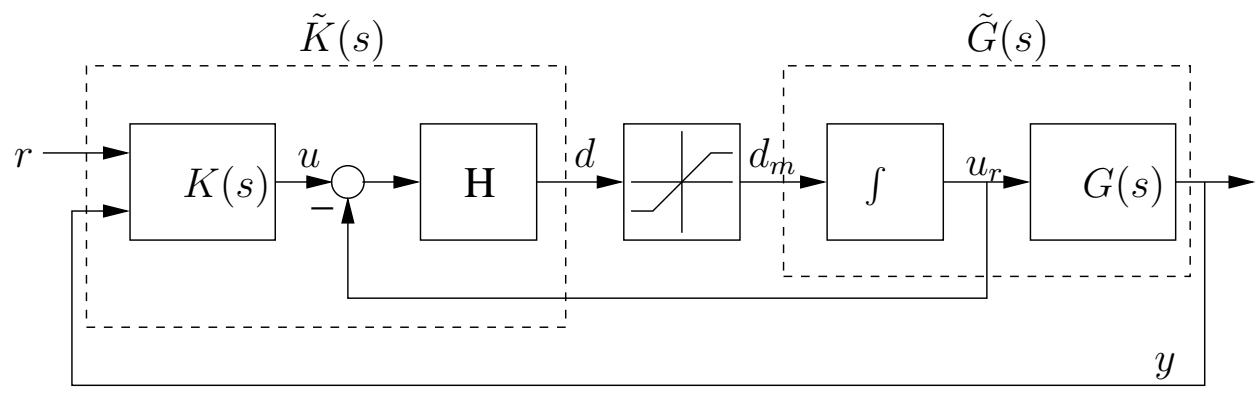

Fig. 3. Equivalent magnitude limited system

When the behaviour of the rate-limit nonlinearity is described by equation (4), the only nonlinearity present in the system depicted in Figure 1, is the saturation nonlinearity internal to the rate-limit. By augmenting the plant and controller as shown in Figure 3, one can translate the rate-limit problem into an equivalent magnitude limit problem. In Figure 3, the "augmented" controller and "augmented" plant are respectively given by

$$
d=\underbrace{H\left[\begin{array}{lll}
K_{1} & K_{2} & -I_{m}
\end{array}\right]}_{\tilde{K}}\left[\begin{array}{c}
r \\
y \\
u_{r}
\end{array}\right] \text { and } \tilde{y}:=\left[\begin{array}{c}
y \\
u_{r}
\end{array}\right]=\underbrace{\left[\begin{array}{c}
G(s) \frac{1}{s} \\
I \frac{1}{s}
\end{array}\right]}_{\tilde{G}(s)} d_{m}
$$

A state-space representation of $\tilde{G}(s)$ is easily derived as

$$
\tilde{G}(s)=\left[\begin{array}{c}
G(s) \frac{1}{s} \\
I \frac{1}{s}
\end{array}\right] \sim\left[\begin{array}{cc|c}
A & B & 0 \\
0 & 0 & I \\
\hline C & D & 0 \\
0 & I & 0
\end{array}\right]=\left[\begin{array}{c|c}
\tilde{A} & \tilde{B} \\
\hline \tilde{C} & 0
\end{array}\right]
$$

The augmented plant now includes dynamics absorbed from the rate-limit model and thus has a larger state-vector $\left[x^{\prime}, x_{r}^{\prime}\right]^{\prime} \in \mathbb{R}^{n_{p}+m}$; its poles are now those of the plant $G(s)$ plus $m$ poles at the origin. The rate-limit problem can thus be thought of as a standard magnitude limit problem with the new "plant", $\tilde{G}$ driven by the signal $d_{m}$ and the new "controller", $\tilde{K}$, producing the "control" signal $d$. 


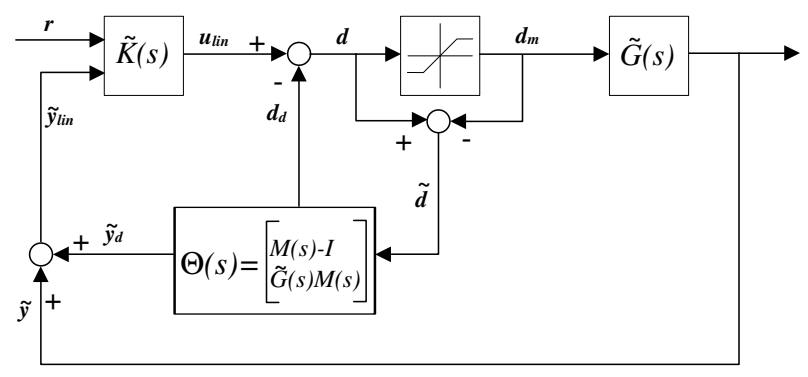

Fig. 4. Equivalent AW Saturation Problem: replace $G(s)$ with $\tilde{G}(s)$

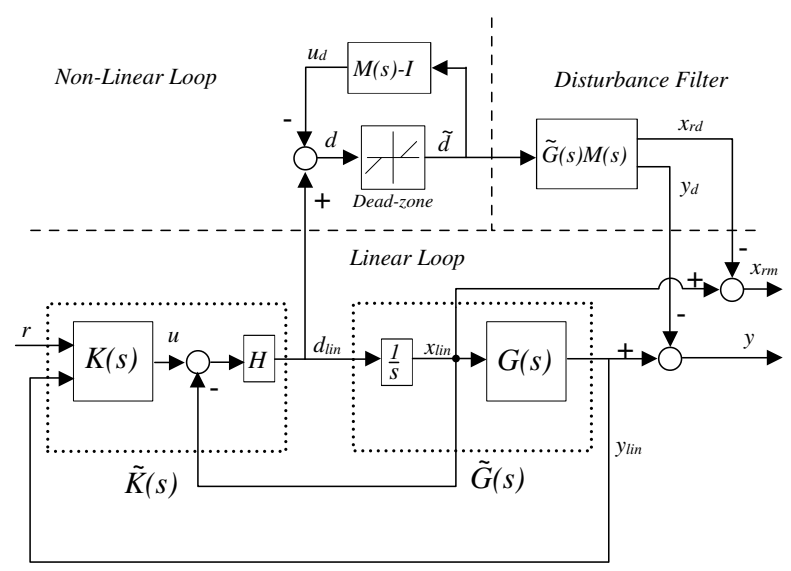

Fig. 5. Equivalent representation of Figure 4

The above implies that rate-saturation problems can be treated in similar ways to magnitude limited problems, and indeed similar AW architectures can be used. Figure 4 shows the architecture used in this paper; the "augmented" plant, $\tilde{G}$, and controller, $\tilde{K}$, are those introduced earlier and the signal $\tilde{y}=\left[\begin{array}{ll}y^{\prime} & x_{r}^{\prime}\end{array}\right]^{\prime} \in \mathbb{R}^{q+m}$ represents the "augmented" measurement from the plant $\tilde{G}$. The anti-windup compensator, $\Theta(s)$, is driven by the signal $\tilde{d}:=d-d_{m}=\operatorname{Dz}(d)$, which is a signal internal to the rate-limit. The outputs from the anti-windup compensator are labeled $d_{d} \in \mathbb{R}^{m}$ and $y_{d} \in \mathbb{R}^{q+m}$ which are injected at the augmented controller's input and output respectively. It is important to mention that this architecture could be difficult to realise for physical rate-limits but as many systems contain software rate-limits to protect the physical rate-limits, it would be straightforward to apply to the software limits.

\section{Anti-windup compensation}

The diagram in Figure 4 is somewhat difficult to interpret and, aside from using the AW compensator $\Theta(s)$ to guarantee the stability of the system, the remaining design goals are unclear. However, with the anti-windup compensator parameterised as

$$
\Theta(s)=\left[\begin{array}{c}
M(s)-I \\
\tilde{G}(s) M(s)
\end{array}\right], \quad M(s) \in \mathcal{R} \mathcal{H}_{\infty}
$$


it is possible to re-draw Figure 4 as Figure 5. In this diagram, the subscripts "lin" denotes a signal which is produced from a purely linear system and the subscript "d" denotes a signal produced by a system driven by the deadzone function, "disturbing" the linear behaviour. This transformation was first proposed in [43,44] (see also [20,40]) and reveals an attractive decoupling of the system into: nominal (unsaturated) linear loop, nonlinear loop and disturbance filter. Two interesting features are evident from the diagram.

i) Assuming that the nominal (unsaturated) loop is stable, then provided $\tilde{G}(s) M(s) \in \mathcal{R} \mathcal{H}_{\infty}$, the nonlinear stability problem reduces to that of guaranteeing stability of the nonlinear loop.

ii) The deviation of the system from nominal linear performance is quantified by the "size" of signal $\tilde{y}_{d}$.

From the above, it follows that the anti-windup problem can be defined in terms of the nonlinear operator $\mathcal{T}_{p}: d_{l i n} \mapsto y_{d}$ [42,31], the "gain" of which dictates how much the linear performance degrades in the presence of (rate) saturation.

\subsection{Problem set-up}

In the magnitude limit case, $G(s) \equiv \tilde{G}(s)$, and therefore, providing that $G(s) \in \mathcal{R} \mathcal{H}_{\infty}$ global stability can be established if global stability of the nonlinear loop can be established for certain $M(s)$; it has been proven (see for example $[13,33]$ ) that global quadratic stability is possible if and only if the nominal linear plant is bounded real, i.e. $\|G(s)\|_{\infty}<\gamma$ for some possitive scalar $\gamma$. The rate-limit case is not quite as simple; even if $G(s)$ is stable, because $\tilde{G}(s)=\left[G(s)^{\prime} / s \quad I / s\right]$, it follows that $\tilde{G}(s) \notin \mathcal{R} \mathcal{H}_{\infty}$ and therefore not bounded real. This implies that we must seek local stability. Additionally, the only way to ensure that the socalled disturbance filter is stable is to introduce zeros in $M(s)$ to "cancel" the unstable poles in $\tilde{G}(s)$. One convenient way to do this is to let $M(s)$ be part of a coprime factorisation of $\tilde{G}(s)=N(s) M(s)^{-1}$. In this case, a state-space realisation for our anti-windup compensator can be given as

$$
\left[\begin{array}{c}
M(s)-I \\
\tilde{G}(s) M(s)
\end{array}\right]=\left[\begin{array}{c}
M(s)-I \\
N(s)
\end{array}\right] \sim\left[\begin{array}{c|c}
\tilde{A}+\tilde{B} F & \tilde{B} \\
\hline F & 0 \\
\tilde{C} & 0
\end{array}\right]
$$

where $F$ is a matrix chosen to ensure $\tilde{A}+\tilde{B} F$ is Hurwitz and is effectively the free parameter in our anti-windup synthesis routine. In this case a state-space realisation of $\mathcal{T}_{p}$, which represents the mapping from our linear "control signal", $d_{l i n}$, to the perturbation to the output, $\tilde{y}_{d}$, can be 
written as

$$
\mathcal{T}_{p} \sim\left\{\begin{aligned}
\dot{\tilde{x}} & =(\tilde{A}+\tilde{B} F) \tilde{x}+\tilde{B} \tilde{d} \\
d_{d} & =F \tilde{x} \\
\tilde{y}_{d} & =\tilde{C} \tilde{x} \\
\tilde{d} & =\mathrm{Dz}\left(d_{\text {lin }}-d_{d}\right)
\end{aligned}\right.
$$

However, this cancelling of the unstable poles of $G(s)$, causes problems in ensuring that the nonlinear loop is globally stable. To circumvent this, we drop the stipulation of global stability and instead seek to ensure that the system is locally exponentially stable. This also makes practical sense, as in most control problems we are not interested in ensuring stability for all possible states; rather we seek stability for a set of states around an operating point. Thus formally we can pose the following problem:

\section{Problem 1: Rate-limit Anti-windup Compensation}

The objective of the rate-limit anti-windup problem is to design a compensator of the form given in equation $(8)$ such that

a) When $d_{\text {lin }} \equiv 0$, the operator $\mathcal{T}_{p}$ is locally exponentially stable; that is $\lim _{t \rightarrow \infty} \tilde{x}(t)=0$ for all $\tilde{x}(t) \in \mathcal{X} \subset \mathcal{A}$.

b) When $\tilde{x}(0) \equiv 0,\left\|\mathcal{T}_{p}\right\|_{i, 2, \mathcal{W}} \leq \gamma$ for some $\gamma>0$ and some set $\mathcal{W}$

As our AW compensator in equation (8) is a function of the state-feedback gain $F$, the above problem essentially reduces to choosing $F$ such that $\mathcal{T}_{p}$ is locally exponentially stable and $\left\|\mathcal{T}_{p}\right\|_{i, 2, \mathcal{W}} \leq \gamma$. Note further that, by the structure of our AW compensator, if $d \in \mathcal{D} \forall t \geq 0$ *, then the AW compensator will remain inactive and linear performance will be not be disturbed. Also, in essence the local exponential stability assumption ensures that if $\operatorname{dist}\left(d_{\text {lin }}, \mathcal{D}\right) \in \mathcal{L}_{2}$ and $d_{\text {lin }} \in \mathcal{W}$, then $\tilde{y}_{d} \in \mathcal{L}_{2}$, ensuring an asymptotic return to linear behaviour if saturation occurs - see [40] or [24] for more details.

\subsection{Local anti-windup}

The concept we make use of to enable local results to be derived is a long-standing tool in constrained input control, perhaps first being introduced in [15] and, since then, used frequently in the anti-windup literature ([45,31] for example).

The idea is as follows. It is well known that the deadzone nonlinearity $\operatorname{Dz}(d) \in \operatorname{Sector}[0, I]$

* The hyper-cuboid $\mathcal{D}=\left[-\bar{d}_{1}, \bar{d}_{1}\right] \times\left[-\bar{d}_{2}, \bar{d}_{2}\right] \times \ldots \times\left[-\bar{d}_{m}, \bar{d}_{m}\right]$ divides the space of the input signal into linear unconstrained region (i.e. $d \in \mathcal{D}$ ) and a saturated region (i.e. $d \notin \mathcal{D}$ ) 
for all $d \in \mathbb{R}^{m}$. Now assume that $d_{i} \leq d_{o, i}$ for some $d_{0, i}$ for all $i$, and define

$$
\mathcal{S}\left(d_{0}\right):=\left\{d \in \mathbb{R}^{m}:-d_{0} \preceq d \preceq d_{0}\right\}
$$

where $d_{0, i}>\bar{d}_{i}$. If this is the case for all time $t>0$, it is evident that $\operatorname{Dz}(d) \in \operatorname{Sector}[0, \mathcal{K}]$ where $\mathcal{K}=\operatorname{diag}\left(\epsilon_{1}, \epsilon_{2}, \ldots, \epsilon_{m}\right)$ and $\epsilon_{i}=\left(d_{o, i}-\bar{d}_{i}\right) / d_{o, i}$. It is possible to simplify this further by letting $\epsilon=\max _{i} \epsilon_{i}$, which implies that $\operatorname{Dz}(d) \in \operatorname{Sector}[0, \epsilon I]$ for all $d \in \mathcal{S}\left(d_{0}\right)$.

Therefore by restricting our attention to $d \in \mathcal{S}\left(d_{0}\right)$ where $d_{0}<\infty$, which in turn implies $\epsilon \in(0,1)$, the deadzone is guaranteed to inhabit a narrower sector than Sector $[0, I]$; that is

$$
d \in \mathcal{S}\left(d_{0}\right) \Rightarrow \operatorname{Dz}(d) \in \operatorname{Sector}[0, \epsilon I]
$$

Note that for any arbitrary large $d_{0}$ there always exists an $\epsilon \in(0,1)$ such that $\operatorname{Dz}(d) \epsilon$ Sector $[0, \epsilon I]$ (in fact, global results are recovered if $d_{0, i}=\infty \quad \forall i \in\{1, \ldots, m\}$, and hence $\operatorname{Dz}(d) \in$ Sector $[0, I])$. Note that by assuming $\operatorname{Dz}(d) \in$ Sector $[0, \epsilon I]$, we are effectively restricting our attention to only a subset of possible signals $d$ and thus results derived on this basis will be necessarily local, rather than global.

\subsection{Region of attraction}

As we are interested in deriving local results, it is desirable to estimate the region in which our proposed anti-windup compensation technique provides local asymptotic stability. In other words, when deriving an anti-windup compensator we also seek a region of attraction (ROA) to accompany it, with it preferably being as large as possible. In general, computation of the region of attraction for nonlinear systems is a nontrivial task and over the years many authors $([39,8,2,12,11,23,38])$ have proposed methods which can be used to estimate the region of attraction for a saturated system.

Quite frequently in saturated systems analysis, an exact description of the region of attraction is not required and here we are content to consider a region of attraction with a crude geometry. In the saturated systems literature (as in [19], [12] for example), an ellipsoidal approximation is typically used,

$$
\mathcal{E}=\left\{\tilde{x} \in \mathbb{R}^{n_{p}+m}: \tilde{x}^{\prime} P \tilde{x}<c\right\} .
$$

The largest such ellipsoid can be estimated (see for example [36], [41]) using a simple formula which will be given later. The salient feature of this type of representation is that it will allow a transparent relationship between AW design parameters and a subset of the region of attraction, $\mathcal{E} \subset \mathcal{X} \subset \mathcal{A}$ to be observed. It must be mentioned that the states of interest when characterising the region of guaranteed stability are the states of our AW compensator $\tilde{x}$, as stability of our linear loop is guaranteed to be global. 


\subsection{Anti-windup solution}

The objective of the anti-windup compensator proposed is to find a $\Theta(s)$ such that

- The map $\mathcal{T}_{p}: d_{l i n} \mapsto y_{d}$ is sufficiently small.

- The nonlinear loop is locally asymptotically stable

We have seen that finding such a $\Theta(s)$ can be reduced to finding an appropriate coprime factorisation $\tilde{G}(s)=N(s) M(s)^{-1}$, and that this, in turn, reduces to finding an appropriate state feedback gain $F$. A systematic way of computing $F$, based on the Riccati equation approach reported in $[33,31]$ is given in the Theorem below.

Theorem 3.1 There exists a full order anti-windup compensator $\Theta=\left[\begin{array}{ll}\Theta_{1}^{\prime} & \Theta_{2}^{\prime}\end{array}\right]^{\prime} \in \mathbb{R}^{(q+2 m) \times m}$, as stated in (8), which solves Problem 1 if there exist matrices $P=P^{\prime}>0, W=\operatorname{diag}\left(w_{1}, \ldots, w_{m}\right)>$ 0 , and positive real scalars $\gamma$ and $\epsilon$ such that the following Riccati equation is satisfied

$$
\tilde{A}^{\prime} P+P \tilde{A}+P \tilde{B}\left(\gamma^{-2} I-2 \frac{(1-\epsilon) W^{-1}}{\epsilon^{2}}\right) \tilde{B}^{\prime} P+\tilde{C}^{\prime} \tilde{C}=0
$$

where the matrix $Z$ must satisfy the following inequality:

$$
Z=\left(2 W-\gamma^{-2}(\epsilon W)^{2}\right)>2 \epsilon W>0
$$

Furthermore, if equation (13) is satisfied, a suitable $\Theta$ is obtained by calculating the matrix $F$ as

$$
F=-\left(\frac{(2-\epsilon) W^{-1}}{\epsilon^{2}}-\gamma^{-2} I\right) \tilde{B}^{\prime} P
$$

A subset of the region of attraction is given by the ellipsoid

$$
\overline{\mathcal{E}}=\left\{\tilde{x} \in \mathbb{R}^{n_{p}+m}: \tilde{x}^{\prime} P \tilde{x}<c_{\max }\right\}
$$

where

$$
c_{\text {max }}=\min _{i} \frac{\bar{d}_{i}^{2} w_{i}^{2}}{(1-\epsilon)^{2}\left(\frac{(2-\epsilon)}{\epsilon^{2}}-\gamma^{-2} w_{i}^{2}\right) B_{i}^{\prime} P B_{i}}
$$

and $w_{i}$ is the $i^{\text {th }}$ diagonal element of $W$ and $B_{i}$ is the $i^{\text {th }}$ column vector of $B$, for some $i \in$ $\{1, \ldots, m\}$.

Proof: A similar Theorem was briefly introduced in [31], although a slightly different proof will be provided here. It is constructed in several stages as detailed below.

i) The main inequality Consider the function 


$$
L:=\frac{d}{d t} \tilde{x}^{\prime} P \tilde{x}+\|\tilde{C} \tilde{x}\|^{2}-\gamma^{2}\left\|d_{l i n}\right\|^{2}+2 \tilde{d}^{\prime} W(\epsilon d-\tilde{d})<0
$$

The final term $2 \tilde{d^{\prime}} W(\epsilon d-\tilde{d})$ arises from the assumption that $d \in \mathcal{S}(d) \forall t \geq 0$ and hence that $2 \tilde{d}^{\prime} W(\epsilon d-\tilde{d}) \geq 0$. Now consider two cases

(1) Assume that $\tilde{x}(0)=0$ and $d_{\text {lin }} \in \mathcal{W}$ where

$$
\mathcal{W}:=\left\{d_{\text {lin }} \in \mathbb{R}^{m}: d \in \mathcal{S}\left(d_{0}\right)\right\}
$$

This implies that $d \in \mathcal{S}\left(d_{0}\right) \forall t \geq 0$ and thus if inequality (18) holds then this implies

$$
\frac{d}{d t} \tilde{x}^{\prime} P \tilde{x}+\|\tilde{C} \tilde{x}\|^{2}-\gamma^{2}\left\|d_{l i n}\right\|^{2}<0
$$

Integration with respect to time from $t=0$ to $t=\infty$ gives

$$
\tilde{x}(\infty)^{\prime} P \tilde{x}(\infty)-\tilde{x}(0)^{\prime} P \tilde{x}(0)+\left\|\tilde{y}_{d}\right\|_{2}^{2}-\gamma^{2}\left\|d_{l i n}\right\|_{2}^{2}<0
$$

which in turn implies that $\left\|\tilde{y}_{d}\right\|_{2}-\gamma\left\|d_{\text {lin }}\right\|_{2}<0$ and hence that $\|\mathcal{T}\|_{i, 2, \mathcal{W}}<\gamma$. i.e. Condition b) of Problem 1 is satisfied.

(2) Next assume that $d_{\text {lin }}=0 \forall t \geq 0$ and that $\tilde{x}(0) \in \mathcal{E}$ where

$$
\mathcal{E}=\left\{\tilde{x} \in \mathbb{R}^{n_{p}+m}: \tilde{x}^{\prime} P \tilde{x} \leq c\right\}
$$

for some $c>0$. Furthermore let $c$ be sufficiently small to ensure that $d \in \mathcal{S}\left(d_{0}\right)$. Then it follows that $2 \tilde{d}^{\prime} W(\epsilon d-\tilde{d}) \geq 0$ and inequality (18) implies that

$$
\frac{d}{d t} \tilde{x}^{\prime} P \tilde{x}=\dot{V}(\tilde{x})<0
$$

and thus $V(\tilde{x})$ is a Lyapunov function for the nonlinear loop in Figure 5, which ensures local asymptotic stability for all $\tilde{x}(0) \in \mathcal{E}$. Thus the system is locally asymptotically stable and hence, if inequality (18) holds, Condition a) of Problem 1 is satisfied.

Thus, it remains for us to prove that satisfaction of inequality (18) is equivalent to the expressions given in the Theorem.

ii) Evaluation of the main inequality. The remainder of the proof shows how we can re-write the cost function $L$, equation (18), as the conditions given in Theorem 3.1. Evaluating equation (18) results in an inequality containing several cross terms in $\tilde{d}, d_{\text {lin }}$, and $\tilde{x}$. Using similar ideas to those proposed in [32], it is possible to address this issue and avoid LMI formulations.

Expanding (18) and substituting $d=d_{l i n}-d_{d}$ gives

$$
L=\tilde{x}^{\prime} \tilde{C}^{\prime} \tilde{C} \tilde{x}-\gamma^{2} d_{l i n}^{\prime} d_{l i n}+\dot{\tilde{x}}^{\prime} P \tilde{x}+\tilde{x}^{\prime} P \dot{\tilde{x}}+2 \tilde{d}^{\prime} \epsilon W d_{l i n}-2 \tilde{d}^{\prime} \epsilon W d_{d}-2 \tilde{d^{\prime}} W \tilde{d}
$$

We now group the cross-product terms in three steps:

(I) Cross-product terms involving $d_{l i n}$ and $\tilde{d}$ are grouped as follows:

$$
-\left[\gamma^{2} d_{l i n}^{\prime} d_{l i n}-2 \tilde{d}^{\prime} \epsilon W d_{l i n}\right]=-\left\|\left(\gamma d_{l i n}-\gamma^{-1} W \epsilon \tilde{d}\right)\right\|^{2}+\gamma^{-2} \tilde{d}^{\prime} \epsilon^{2} W^{2} \tilde{d}
$$


Combining the above with (24), a cost function containing no cross-product terms between $d_{l i n}$ and $\tilde{d}$ is obtained. Using equation (9) to expand $\dot{\tilde{x}}$ and noticing that $d_{d}=F \tilde{x}$ :

$$
\begin{aligned}
L= & \tilde{x}^{\prime}\left(\tilde{C}^{\prime} \tilde{C}+\tilde{A}^{\prime} P+P \tilde{A}+2 F^{\prime} \tilde{B}^{\prime} P\right) \tilde{x}+2 \tilde{x}^{\prime}\left(P \tilde{B}-\epsilon F^{\prime} W\right) \tilde{d} \\
& -\tilde{d}^{\prime} Z \tilde{d}-\left\|\gamma d_{l i n}-\gamma^{-1} W \epsilon \tilde{d}\right\|^{2}
\end{aligned}
$$

where $Z=2 W-\gamma^{-2} \epsilon^{2} W^{2}$

(II) Cross-Product terms involving $\tilde{d}$ and $\tilde{x}$ are grouped as follows:

$$
\begin{aligned}
-\left[\tilde{d}^{\prime} Z \tilde{d}-2 \tilde{x}^{\prime}\left(\tilde{B}^{\prime} P-\epsilon W F\right)^{\prime} \tilde{d}\right]= & -\left\|\left(Z^{\frac{1}{2}} \tilde{d}-Z^{-\frac{1}{2}}\left(\tilde{B}^{\prime} P-\epsilon W F\right) \tilde{x}\right)\right\|^{2}+ \\
& \tilde{x}^{\prime}\left(\tilde{B}^{\prime} P-\epsilon W F\right)^{\prime} Z^{-1}\left(\tilde{B}^{\prime} P-\epsilon W F\right) \tilde{x}
\end{aligned}
$$

Note that $Z$ must be positive definite in order to have a well posed problem. This condition arises from the necessity of making the quadratic term

$$
-\left\|\left(Z^{\frac{1}{2}} \tilde{d}-Z^{-\frac{1}{2}}\left(\tilde{B}^{\prime} P-\epsilon W F\right) \tilde{x}\right)\right\|^{2}
$$

negative for any pair $(\tilde{d}, \tilde{x})$. It can easily be shown that if $Z<0$, this is not always guaranteed. By replacing this new group of terms, a new cost function is obtained. This cost function has no cross-product terms between $\tilde{d}$ and $\tilde{x}$.

$$
L \leq \tilde{x}^{\prime}\left(\tilde{C}^{\prime} \tilde{C}+\tilde{A}^{\prime} P+P \tilde{A}+2 F^{\prime} \tilde{B}^{\prime} P+P \tilde{B} Z^{-1} \tilde{B}^{\prime} P-2 F^{\prime} W \epsilon Z^{-1} \tilde{B}^{\prime} P+F^{\prime} W \epsilon Z^{-1} \epsilon W F\right) \tilde{x}
$$

(III) Terms involving $F$ and $F^{\prime} F$ are grouped:

$$
\begin{array}{r}
F^{\prime} W \epsilon Z^{-1} \epsilon W F+2 P \tilde{B}\left(I-Z^{-1} \epsilon W\right) F=\left\|Z^{-\frac{1}{2}}\left(\epsilon W F+\left(Z \epsilon^{-1} W^{-1}-I\right) \tilde{B}^{\prime} P\right)\right\|^{2} \\
-P \tilde{B}\left(Z \epsilon^{-1} W^{-1}-I\right)^{\prime} Z^{-1}\left(Z \epsilon^{-1} W^{-1}-I\right) \tilde{B}^{\prime} P
\end{array}
$$

Thus it follows that our cost function (18) can be written as

$$
L=L_{a}+L_{b}+L_{c}
$$

where

$$
\begin{aligned}
L_{a}= & \tilde{x}^{\prime}\left(\tilde{C}^{\prime} \tilde{C}+\tilde{A}^{\prime} P+P \tilde{A}+P \tilde{B} Z^{-1} \tilde{B}^{\prime} P-\right. \\
& \left.P \tilde{B}\left(Z \epsilon^{-1} W^{-1}-I\right)^{\prime} Z^{-1}\left(Z \epsilon^{-1} W^{-1}-I\right) \tilde{B}^{\prime} P\right) \tilde{x} \\
L_{b}= & \left\|Z^{-\frac{1}{2}}\left(\epsilon W F+\left(Z \epsilon^{-1} W^{-1}-I\right) \tilde{B}^{\prime} P\right) \tilde{x}\right\|^{2} \\
L_{c}= & -\left\|Z^{\frac{1}{2}} \tilde{d}-Z^{-\frac{1}{2}}\left(\tilde{B}^{\prime} P-\epsilon W F\right) \tilde{x}\right\|^{2}-\left\|\gamma d_{l i n}-\gamma^{-1} W \epsilon \tilde{d}\right\|^{2}
\end{aligned}
$$

Equation (30) comprises three terms; the last, $L_{c}$, is a negative definite quadratic term. Therefore if the first two terms can be set to zero, then $L()<$.0 . Setting the second term, $L_{b}$, and noting that $Z>0$, yields:

$$
\epsilon W F+\left(Z \epsilon^{-1} W^{-1}-I\right) \tilde{B}^{\prime} P=0
$$


Using the expression for $Z\left(Z=2 W-\gamma^{-2} \epsilon^{2} W^{2}\right)$ yields the following condition for the gain matrix $F$ :

$$
F=-\left(\frac{(2-\epsilon) W^{-1}}{\epsilon^{2}}-\gamma^{-2}\right) \tilde{B}^{\prime} P
$$

where $P=P^{\prime}>0$ comes from solving the Ricatti equation which makes $L_{a}=0$ :

$$
\begin{array}{ccc} 
& \tilde{A}^{\prime} P+P \tilde{A}+C^{\prime} C+P \tilde{B} Z^{-1} \tilde{B}^{\prime} P-P \tilde{B}\left(Z \epsilon^{-1} W^{-1}-I\right)^{\prime} Z^{-1}\left(Z \epsilon^{-1} W^{-1}-I\right) \tilde{B}^{\prime} P=0 \\
\Leftrightarrow & \tilde{A}^{\prime} P+P \tilde{A}+C^{\prime} C+P \tilde{B}\left[Z^{-1}-\left(Z \epsilon^{-1} W^{-1}-I\right) Z^{-1}\left(Z \epsilon^{-1} W^{-1}-I\right)\right] \tilde{B}^{\prime} P & =0 \\
\Leftrightarrow & \tilde{A}^{\prime} P+P \tilde{A}+C^{\prime} C+P \tilde{B}\left[-\epsilon^{-2} W^{-2} Z+2 \epsilon^{-1} W^{-1}\right] \tilde{B}^{\prime} P & =0
\end{array}
$$

Equation (13) is then obtained by substituting $Z=2 W-\gamma^{-2} \epsilon^{2} W^{2}$ into equation (38).

iii) Calculation of the region of attraction. In part i) it was shown that the system is locally asymptotically stable in a set $\mathcal{E} \subset \mathcal{X}$ where $\mathcal{X}$ is a subset of the region of attraction. As mentioned earlier, we estimate ellipsoidal subsets of the region of attraction and in particular are interested in calculating the largest such ellipsoid, $\overline{\mathcal{E}}$ which has the following form

$$
\overline{\mathcal{E}}=\left\{\tilde{x} \in \mathbb{R}^{n_{p}+m}: \tilde{x}^{\prime} P \tilde{x}<c_{\max }\right\} .
$$

For the estimation of the region of attraction it is assumed that $d_{l i n}=0$ and, to ensure that the deadzone is within a reduced sector, that the states $\tilde{x}$ belong to the set

$$
\mathcal{X}=\left\{\tilde{x} \in \mathbb{R}^{n_{p}+m}: d \in \mathcal{S}\left(d_{0}\right) \forall t \geq 0\right\} \subset \mathcal{A}
$$

Thus to calculate an ellipsoidal region of attraction we seek the largest set such that

$$
\tilde{x} \in \overline{\mathcal{E}} \Rightarrow d \in \mathcal{S}\left(d_{0}\right) \forall t \geq 0
$$

or equivalently we seek the largest set

$$
\overline{\mathcal{E}}=\left\{\tilde{x} \in \mathbb{R}^{n_{p}+m}: \tilde{x}^{\prime} P \tilde{x}<c_{\max }, \quad|d|=|F \tilde{x}| \preceq d_{0}\right\}
$$

Thus if $\tilde{x} \in \overline{\mathcal{E}}$ it follows that $d \in \mathcal{S}\left(d_{0}\right)$ and $\operatorname{Dz}(d) \in \operatorname{Sector}[0, \epsilon I]$; hence the system is locally stable. To calculate $\overline{\mathcal{E}}$, we follow a method used in, for example, [36], [41]. The largest ellipsoid region of attraction can be posed a maximisation problem:

$$
\max _{\tilde{x}} \tilde{x}^{\prime} P \tilde{x} \quad \text { s.t. } \quad|F \tilde{x}| \preceq d_{0}
$$

which is equivalent to

$$
\max _{\tilde{x}} \tilde{x}^{\prime} P \tilde{x} \quad \text { s.t. } \quad\left|F_{i} \tilde{x}\right|<d_{0, i} \forall i \in\{1, \ldots, m\}
$$

where, $F_{i}$ is the $i^{\text {th }}$ row of $F$. The largest such ellipsoid can then be calculated using

$$
c_{\max }=\min _{i} \frac{d_{0, i}^{2}}{F_{i} P^{-1} F_{i}^{\prime}}
$$


Substituting for $F$ yields

$$
c_{\max }=\frac{\bar{d}_{i}^{2} w_{i}^{2}}{(1-\epsilon)^{2}\left(\frac{(2-\epsilon)}{\epsilon^{2}}-\gamma^{-2} w_{i}^{2}\right) B_{i}^{\prime} P B_{i}}
$$

which completes the proof.

Remark 2: It can be seen that as $\epsilon$ gets close to unity, the size of $c_{\text {max }}$, and hence the size of $\overline{\mathcal{E}}$ increases, for fixed $\gamma$ and $W$. Thus by making $\epsilon$ arbitrarily close to unity, the size of the region of attraction can be made arbitrarily large for some appropriate choice of $\gamma$ and $W$.

\subsection{Improved tuning procedure}

The main issue in using Theorem 3.1 for anti-windup synthesis is that the Riccati equation (13) is not guaranteed to be solvable for arbitrary $\gamma, \epsilon$ and $W$ and hence the design could be complex. However, using equation (14), which imposes the inequality constraint $Z>0$, it is possible re-write Theorem 3.1 in a manner where a solution to the Riccati equation (13) is always guaranteed.

Corollary 1 There exists a full order anti-windup compensator $\Theta=\left[\begin{array}{ll}\Theta_{1}^{\prime} & \Theta_{2}^{\prime}\end{array}\right]^{\prime} \in \mathbb{R}^{(q+2 m) \times m}$ which solves strongly the anti-windup problem if there exists a matrix $P=P^{\prime}>0$, and positive real scalars $\epsilon \in(0,1)$, and $\rho>0$ such that the following Riccati equation is satisfied

$$
\tilde{A}^{\prime} P+P \tilde{A}-\rho P \tilde{B} \tilde{B}^{\prime} P+\tilde{C}^{\prime} \tilde{C}=0
$$

Furthermore, if equation (47) is satisfied, a suitable $\Theta$ achieving $\|\mathcal{T}\|_{i, 2, \mathcal{W}}<\gamma$ is obtained by calculating the matrix gain $F$ as follows:

$$
F=-(1-\epsilon)^{-1} \rho \tilde{B}^{\prime} P
$$

where $\gamma=\sqrt{\frac{1}{\rho}}$. An estimate the region of attraction is given by the ellipsoid

$$
\overline{\mathcal{E}}=\left\{\tilde{x}: \tilde{x}^{\prime} P \tilde{x} \leq c_{\max }\right\} \quad \text { where } \quad c_{\max }=\max _{i}\left\{\frac{\bar{d}_{i}^{2}}{\rho^{2} \tilde{B}_{i}^{\prime} P \tilde{B}_{i}}\right\}
$$

Proof: The proof will show how, by re-defining certain parameters, the expressions in Theorem 3.1 can be re-written as those in Corollary 1. As before, $\gamma$ and $\epsilon$ are assumed to be positive scalars, and $W$ is chosen to be $W=\omega I, \quad \omega>0$. First note that using inequality (14), we 
obtain a bound on $\gamma^{-2}$ as

$$
\gamma^{-2} I<2 \frac{(1-\epsilon)}{\epsilon^{2}} W^{-1} \Rightarrow \exists k \in(0,2) \quad \text { s.t. } \quad \gamma^{-2}=k \frac{(1-\epsilon)}{\epsilon^{2}} \omega^{-1}
$$

The extra term $k$ is included in order to allow us to rewrite the inequality as an equality. Substituting this into equation (13) yields the Riccati equation

$$
\tilde{A}^{\prime} P+P \tilde{A}-P \tilde{B} R \tilde{B}^{\prime} P+\tilde{C}^{\prime} \tilde{C}=0
$$

where $R$ is defined as $R=(2-k) \frac{(1-\epsilon)}{\epsilon^{2}} \omega^{-1} I$. This allows us to replace $R$ by $R=\rho I$ where

$$
\rho=(2-k) \frac{(1-\epsilon)}{\epsilon^{2}} \omega^{-1}
$$

which yields Riccati equation (47) in Corollary 1. Similarly using the expressions for $\rho$ and $\gamma^{-2}$ in equation (15) allows us to write

$$
F=-\left(R+\frac{W^{-1}}{\epsilon}\right) \tilde{B}^{\prime} P F=-(1-\epsilon)^{-1}\left(1+\frac{(k-1) \epsilon}{2-k}\right) \rho \tilde{B}^{\prime} P
$$

Similarly, substituting this expression for $F$ into equation (45) allows a $c_{\max }$ to be calculated as

$$
c_{\max }=\max _{i}\left\{\frac{\bar{d}_{i}^{2}}{\rho^{2}\left(1+\frac{(k-1) \epsilon}{2-k}\right)^{2} \tilde{B}_{i}^{\prime} P \tilde{B}_{i}}\right\}
$$

Note that the expression for the Riccati equation is simpler than before (it is only a function of $\rho$ ) but the expression for the $\gamma$ and the region of attraction are still not clear. However, note from equation (50), the effect of $k$ can be offset to some extent by tuning $W=\omega I$ appropriately. Thus, if one simply fixes $k=1$, it follows that the expressions for $F$ and $c_{\max }$ reduce to those given in the Theorem

Remark 3: The usefulness of Corollary 1 is in its ease of tuning: the three parameters $(\epsilon \in$ $(0,1), \omega>0$ and $\gamma>0)$ of Theorem 3.1 have been replaced by just two $(\epsilon \in(0,1)$ and $\rho>0)$. Furthermore, the Riccati equation in Corollary 1 is just a function of $\rho$ and is just a standard LQR-type Riccati equation which will always have a solution $P=P^{\prime}>0$ as $(\tilde{A}, \tilde{B}, \tilde{C})$ are assumed stabilisable and detectable

This new formulation is appealing because the Riccati equation is purely a function of $\rho$ and an explicit bound on the local $\mathcal{L}_{2}$ gain is given by $\gamma$. Another of the interesting features of this new simplified strategy is that it allows precise mathematical conclusions to be drawn about the trade-off between the local performance level and the region of attraction: it can be 
observed that equations (52) and (54) embody the trade-off between performance and region of attraction as they both involve $\rho$. In particular it is interesting to see how the region of attraction's size alters as a function of $\rho$. Note that the region of attraction could equivalently be expressed as

$$
\begin{aligned}
\overline{\mathcal{E}} & =\left\{\tilde{x} \in \mathbb{R}^{n_{p}+m}: \tilde{x}^{\prime} \rho E(\rho) \tilde{x} \leq 1\right\}, \\
E(\rho) & :=\min _{i} \rho P(\rho) \frac{\rho \tilde{B}_{i}^{\prime} P \tilde{B}_{i}}{\bar{d}_{i}^{2}}=\min _{i} \rho P(\rho) \frac{\rho P_{i i}(\rho)}{\bar{d}_{i}^{2}}
\end{aligned}
$$

It is difficult to characterise the size of this ellipsoid, but one way of doing so is by the sum of the length of its semi-axes, which is equal to the trace of $E^{-1}(\rho)$, which can be written as

$$
\operatorname{tr}\left[E^{-1}(\rho)\right]=\max _{i} \bar{d}_{i}^{2} \operatorname{tr}\left\{[\rho P(\rho)]^{-1}\left[\rho P_{i i}(\rho)\right]^{-1}\right\}
$$

So the key parameter which determines the "size" of the region of attraction is the matrix $\rho P(\rho)$. We will establish how this varies as a function of $\rho$. First, multiply equation (47) by $\rho$ to get

$$
\tilde{A}^{\prime} P \rho+\rho P \tilde{A}-\rho P \tilde{B} \tilde{B}^{\prime} P \rho+\rho \tilde{C}^{\prime} \tilde{C}=0
$$

Defining $Q(\rho):=P(\rho) \rho>0$ we now have

$$
\tilde{A}^{\prime} Q(\rho)+Q(\rho) \tilde{A}-Q(\rho) \tilde{B} \tilde{B}^{\prime} Q(\rho)+\rho \tilde{C}^{\prime} \tilde{C}=0 .
$$

Now if $\left(\rho^{\frac{1}{2}} \tilde{C}, \tilde{A}\right)$ is detectable, it follows $[28,39,16]$ that

$$
\frac{d Q(\rho)}{d \rho}>0, \quad \lim _{\rho \rightarrow 0} Q(\rho)=0
$$

From this it also follows that $d Q_{i i}(\rho) / d \rho>0$. Thus $P(\rho) \rho=Q(\rho)$ and $\rho P_{i i}(\rho)=Q_{i i}(\rho)$ are monotonically increasing functions of $\rho$. Hence $\operatorname{tr}\left[E^{-1}(\rho)\right]=\max _{i} \bar{d}_{i}^{2} \operatorname{tr}\left[Q^{-1}(\rho) Q_{i i}^{-1}(\rho)\right]$, is a monotonically decreasing function of $\rho$ which implies that as $\rho$ increases, the "size" of the region of attraction decreases. Furthermore, in the limit as $\rho$ tends to zero $(\epsilon \rightarrow 1$ i.e. global results), the region of attraction becomes the whole state-space.

Note also that we can write $F$ as

$$
F=\frac{-1}{1-\epsilon} \tilde{B}^{\prime} Q(\rho),
$$

and thus we can see that as $\rho$ becomes smaller, $F$ becomes smaller (in the sense that its norm reduces) and as $\rho \rightarrow 0, F \rightarrow 0$ and we recover the IMC solution; this also corresponds to 
$\gamma=\infty$. In other words, we have the "worst" performance for the IMC AW case (which is not surprising since $\tilde{G}(s)$ is not bounded real). In conclusion we can see that

$$
\gamma \propto \frac{1}{\sqrt{\rho}} \text { and }|\overline{\mathcal{E}}| \propto \frac{1}{\rho}
$$

meaning that there is a clear trade-off between minimising the local $\mathcal{L}_{2}$ gain and maximising the size of the region of attraction.

Remark 4 The tuning procedure presented above states that for a given $\rho$ - and therefore for a given performance bound and region of attraction - there exists a family of compensator gains, $F$, parametrised by $\epsilon \in(0,1)$. The parameter $\epsilon$ can be seen to play a similar role to the free parameter in the low-high-gain design of [19] and one may expect some performance enhancement when $\epsilon$ is chosen close to unity. However, it is difficult to specify precisely what this performance enhancement is. Experience suggests that when $\epsilon$ is chosen close to unity (i.e. $F$ is "high gain") noticeably better time domain performance is observed when the states are close to the origin, but that this can worsen dramatically for states closer to the edge (but within) the ROA. When $\epsilon$ is chosen closer to zero (i.e. $F$ is "low gain"), the difference between the small and large signal performance of the compensators does not seem so noticeable and appears to allow the compensators to preserve stability for regions beyond the guaranteed ROA.

\section{Example}

In this section the effectiveness of the results are shown through a well known academic example. Consider the problem of a missile auto-pilot as presented in [33,26,25], which is a third order two-input-two-output dynamic linear system. As required, the feedback interconnection of the plant and controller when no rate-limits are present is internally stable and well posed. However the "real" nonlinear system has actuators with rate constraints of $\pm 3[\mathrm{deg} / \mathrm{s}]$ imposed in both channels.

Figure 6(a) shows the nominal linear response of the missile for a pulse $r=[5-5]$. Notice the decoupled outputs and swift but well-damped rise-times in both channels. However, it must be mentioned that the rate of the control signal exceeds the imposed limits and consequently the system with rate-limited actuators could exhibit a loss in performance and perhaps stability. Figure 6(b) confirms this and shows clearly how the system looses its decoupling and tracking properties when rate-limits are introduced.

To limit the performance degradation during periods of rate saturation an AW compensator is introduced. As mentioned earlier, for the rate saturated case, the task of choosing appropriate values of the design parameters presented in Theorem 3.1 is not as simple as in [32], but the approach is still intuitive. The designer's task can be simplified by using the tuning algorithm proposed in Section 3.5; we will concentrate on this approach. 


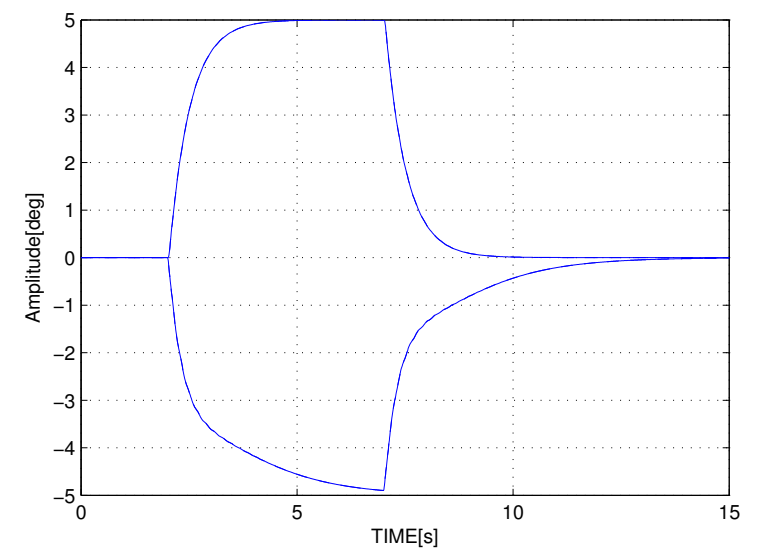

output signal

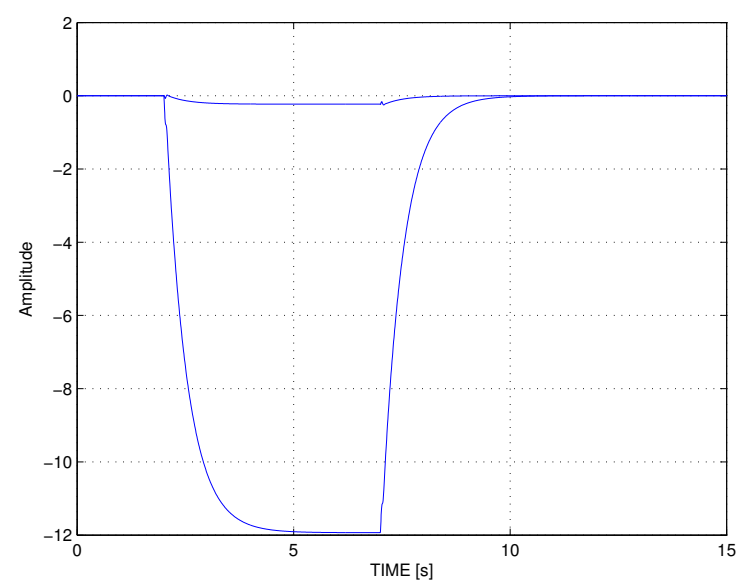

control signal

(a) Nominal Response

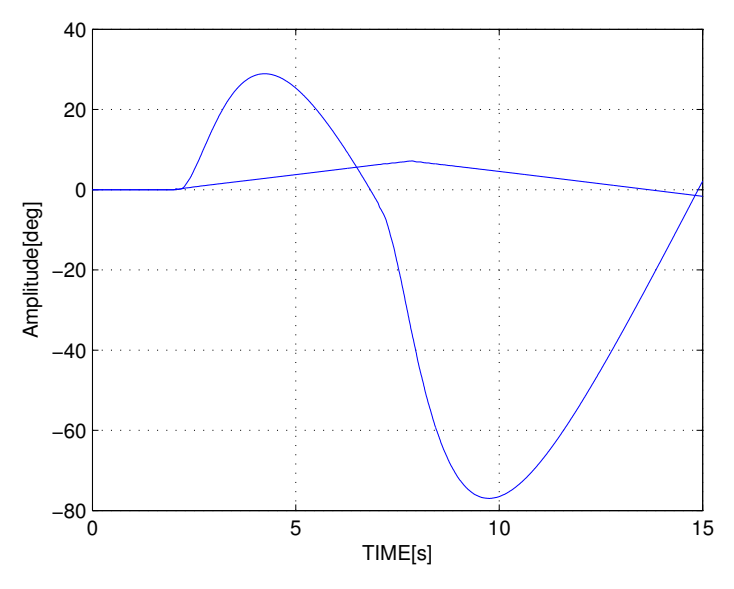

output signal

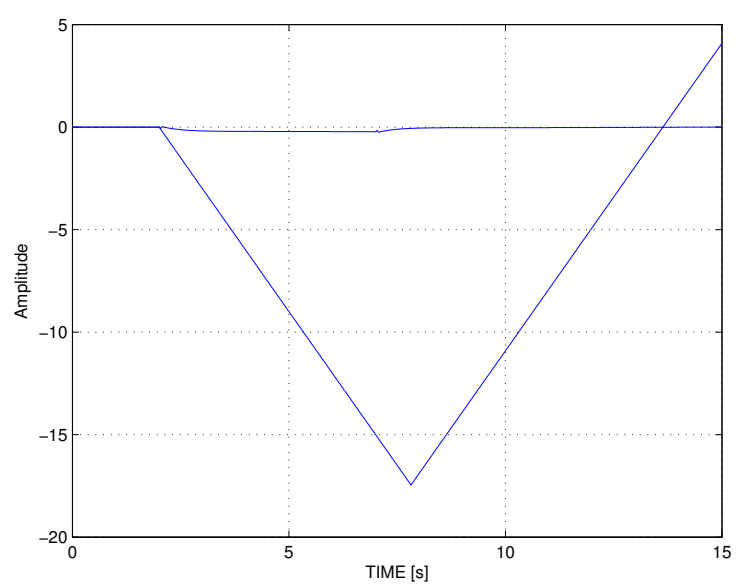

control signal

(b) Rate Saturated Response with no AW

Fig. 6. System Response

Corollary 1 provides a simple way of characterising the performance level and stability region of the AW compensator. Notice that the available parameters are $\rho$ and $\epsilon$, where $\rho$ gives a measure of nonlinear performance and $\epsilon$ defines the polyhedral set of allowed signals $d$, i.e. $\mathcal{S}\left(d_{0}\right)=\left\{d:-d_{0} \preceq d \preceq d_{0}\right\}$. The main goal of our design is to synthesize an AW compensator which guarantees stability in as large as set as possible while preserving performance as much as possible. As shown earlier, in order to achieve good performance (i.e. $\left\|\mathcal{T}_{p}\right\|_{i, 2, \mathcal{W}}$ small), it is desirable to choose $\rho$ large; from equation (54) it can be concluded that for an enlarged region of attraction $\rho$ must be small. In other words, the trade-off between performance and region of attraction is encapsulated in the design parameter $\rho$.

The main issue with the approach used in this paper is that it is local in nature: performance and stability guarantees are ensured only if $d \in \mathcal{S}\left(d_{0}\right)$. On the other hand, the conditions presented are only sufficient, and a system which does not guarantee $d \in \mathcal{S}\left(d_{0}\right)$ may still be stable within a large region of attraction (or perhaps the whole state space). Even if we assume 


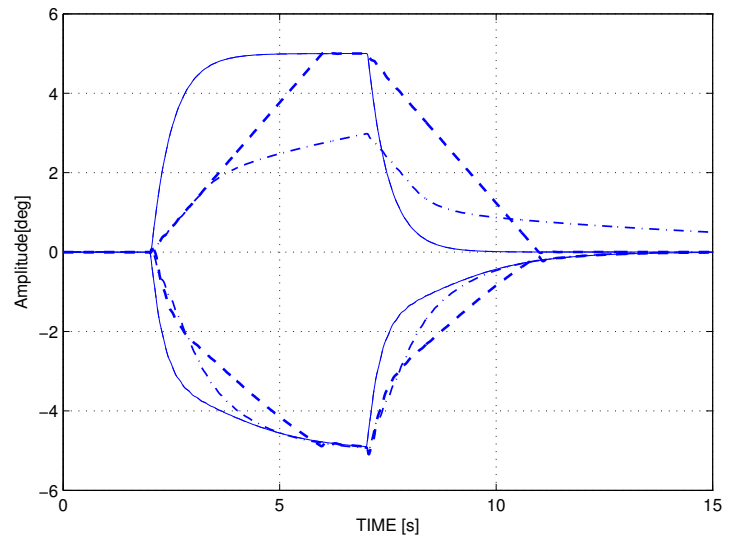

output signal

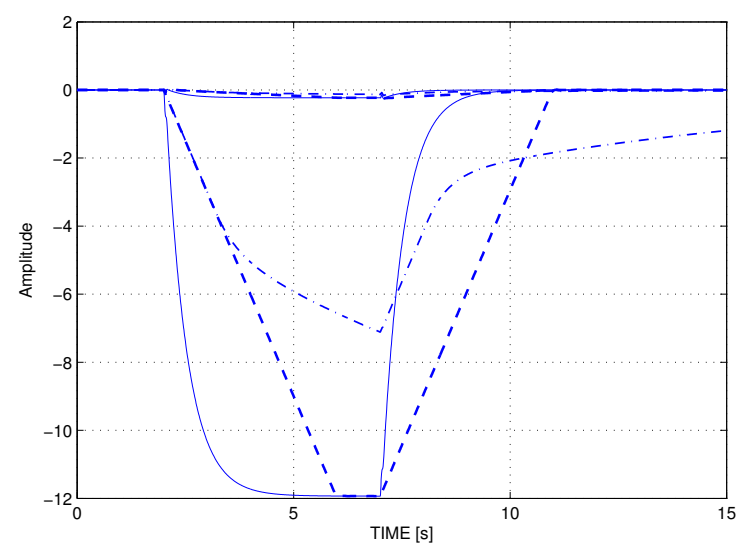

control signal

(a) Reference 5[deg]

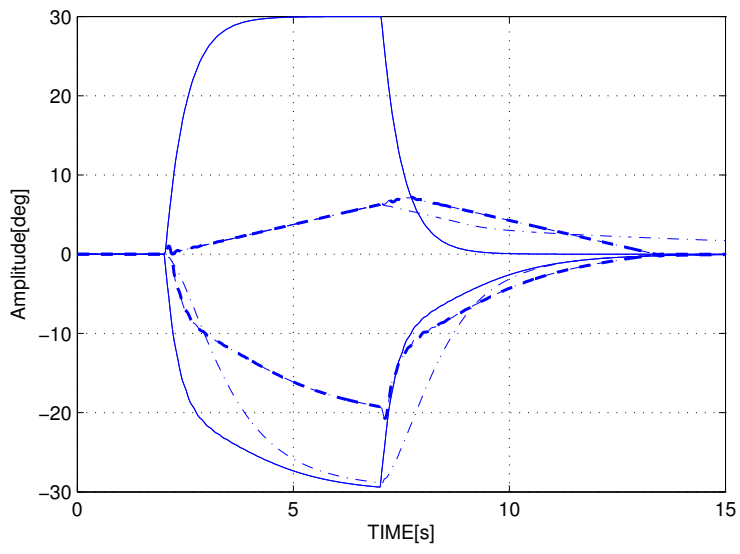

output signal

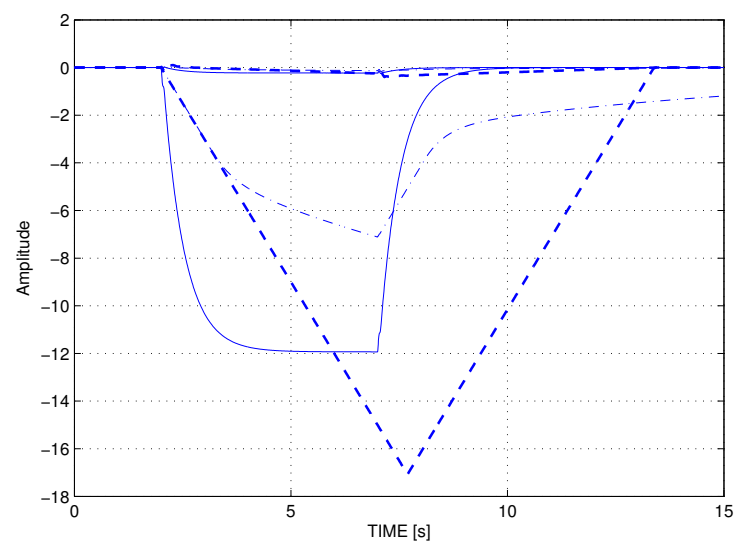

control signal

(b) Reference 30[deg]

Fig. 7. Rate-limited response of missile: nominal linear (solid), full-order AW $\rho_{\text {low }}$ (dash-dot), full-order AW $\rho_{\text {high }}$ (dash)

that the AW compensator can guarantee global (or semiglobal) stability, degradation of the closed-loop compensated system's performance may still be present as the norm bound of the map $\mathcal{T}_{p}$ no longer holds. In fact, after some analysis, it can be inferred that as the system moves away from the pre-established ROA, the gain $\left\|\mathcal{T}_{p}\right\|_{i, 2, \mathcal{W}}$ increases, yielding an increase on the system's deviation from linear dynamics and hence a degradation of the prescribed nonlinear performance.

To illustrate the design of AW compensators using Corollary 1, two compensators are designed: $\epsilon=0.9$ is fixed and $\rho$ takes a values of $\rho_{\text {low }}=10^{-4}$ and $\rho_{\text {high }}=10$ for the "low" and "high" performance compensators respectively.

Figure 7(a) depicts missile responses obtained with the $\rho_{\text {low }}$ and $\rho_{\text {high }}$ AW compensators respectively and a reference pulse with amplitude of $\pm 5[\mathrm{deg}]$. Clearly in both cases the system's response is enhanced by the use of AW compensation: rate saturation levels are reduced, 
tracking properties are retained and return to linear behaviour is well behaved. It is somewhat more difficult to conclude which compensator delivers the best performance; for the low AW compensator the second channel exhibits fast tracking performance while the first channel is sluggish. On balance however, one might say that the "high performance" compensator does indeed perform better for this small input demand.

Figure 7(b) illustrates the missile responses obtained with the two compensators when the reference demands are increased to $\pm 30[\mathrm{deg}]$. Such a large reference demand causes the system to depart from its guaranteed region of stability (i.e. $d \notin \mathcal{S}\left(d, d_{o}\right)$ ) and while stability is not lost, performance does degrade. As the region of stability for the high performance compensator is smaller than that of the low performance compensator, it is not surprising that it's performance deteriorates more. Although both compensators suffer a lack of tracking ability in the first channel (due to the restricted rate and short duration of the pulse demand), the low performance compensator retains its tracking ability in the second channel and exhibits a reduced phase-lag in the first channel. However, the high gain compensator is unable to preserve this tracking performance and the second channel fails to follow that of the linear system well. In conclusion, this example it appears preferable to accept a loss of small-signal performance in exchange for better preservation of large-signal performance.

\section{Conclusion}

This paper has proposed an anti-windup scheme for systems with rate-limited actuators. The proposed synthesis algorithms have been constructed using ideas which are reasonably well known in the AW literature, although the Riccati-based solution is novel and superior from a computational perspective. A key feature of the proposed AW algorithms is that they are fairly easy to tune and feature a parameter, $\rho$, which captures the trade-off between local performance and the size of a subset of the region of attraction.

The proposed algorithms have been demonstrated on a simple example, but their real use is envisaged for complex, high order systems where LMI-based methods may have difficulty. These algorithms were used for the design of the AW compensators for investigating ratelimiting on the ATTAS aircraft. Details of the flight tests are reported in [4-6] but, broadly speaking, it was found that the methods described within this paper are useful for designing AW compensators for complex, practical systems.

\section{References}

[1] Anon. Why the grippen crashed. Aerospace America, page 11, 1994.

[2] C. Barbu, R. Reginatto, A.R. Teel, and L. Zaccarian. Anti-windup design for manual flight control. Proc. American Control Conference, pages 3186-3190, 1999.

[3] C. Bolkcom. V-22 osprey tilt-rotor aircraft. CRS Report for Congress, page Code RL31384, 2005. 
[4] O. Brieger, M. Kerr, D. Leissling, I. Postlethwaite, J. Sofrony, and M.C. Turner. Flight testing of a rate-compensation scheme on the ATTAS aircraft. Proc. DGLR Conference, 2006.

[5] O. Brieger, M. Kerr, D. Leissling, I. Postlethwaite, J. Sofrony, and M.C. Turner. Anti-windup compensation of rate saturation in an experimental aircraft. In Proc. American Cont. Conf., New York, 2007.

[6] O. Brieger, M. Kerr, D. Leissling, I. Postlethwaite, J. Sofrony, and M.C. Turner. Flight testing of a rate saturation compensation scheme on the attas aircraft. Aerospace Science and Technology, to appear, 2008.

[7] Y.Y. Cao and Z. Lin. An anti-windup design for polytopic systems by a parameter-dependent lyapunov function approach. International Journal of Systems and Science, 37(2):129-139, 2006.

[8] Y.Y. Cao, Z. Lin, and D.G. Ward. An anti-windup approach to enlarging the domain of attraction for linear systems subject to input sauration. IEEE Trans. Automatic Cotrol, 47(1):140-145, 2002.

[9] S. Crawshaw. Global and local analysis of coprime-factor based anti-windup for stable and unstable plants. Proc. European Control Conference, 2003.

[10] C. Edwards and I. Postlethwaite. Anti-windup and bumpless transfer schemes. Automatica, 34(2):199-210, 1998.

[11] J.M. Gomes da Silva Jr., S. Tarbouriech, and G. Garcia. Local stabilisation of linear systems under amplitude and rate saturating actuators. IEEE Transactions on Automatic Control, 48(5):842-847, 2003.

[12] J.M. Gomes da Silva Jr., S. Tarbouriech, and R. Reginatto. Analysis of regions of stability for linear systems with saturating inputs through an anti-windup scheme. Proc. IEEE Conference on Control Applications, 2002.

[13] G. Grimm, J. Hatfield, I. Postlethwaite, A.R. Teel, M.C. Turner, and L. Zaccarian. Anti-windup for stable linear systems with input saturation: an LMI based synthesis. IEEE Transactions on Automatic Control, 48(9):1509-1525, 2003.

[14] G. Herrmann, M.C. Turner, and I. Postlethwaite. Pratical implementation of a novel anti-windup scheme in a hdd-dual-stage servo-system. IEEE/ASME Transactions on Mechatronics, 9(3):580592, 2004.

[15] H. Hindi and S. Boyd. Analysis of linear systems with saturation using convex optimization. In 37th IEEE Conference on Decision and Control, pages 903-908, Tampa, Florida, USA, 1998.

[16] T. Hu, A.R. Teel, and L. Zaccarian. Regional anti-windup compensation for linear systems with input saturation. In American Control Conference, Portland (OR), USA, pages 3397-3402, 2005.

[17] M. Kerr, E. Villota, and S. Jayasuriya. Robust anti-windup design for input constrained SISO systems. Proc. 8th Int. Symposium on QFT and Robust Frequency Domain Methods., 2007.

[18] M.V. Kothare, P.J. Campo, M. Morari, and C.N. Nett. A unified framework for the study of antiwindup designs. Automatica, 30(12):1869-1883, 1994.

[19] Z. Lin, R. Mantri, and A. Saberi. Semi-global output regulation for linear systems subject to input saturation - a low and high gain design. Control Theory and Advanced Technology, 10(4):22092232, 1998. 
[20] S. Miyamoto and G. Vinnicombe. Robust control of plants with saturation nonlinearity based on comprime factor representations. Proc. IEEE Conf. on Decision and Control, pages 2838-2840, 1996.

[21] E.F. Mulder, M.V. Kothare, and M. Morari. Multivariable anti-windup controller synthesis using linear matrix inequalities. Automatica, 37(9):1407-1416, 2001.

[22] C. Pittet, S. Tarbouriech, and C. Burgat. Stability regions for linear systems with saturating control via Circle and Popov criteria. Proc. IEEE Conference on Decision and Control, 5:4518-4523, 1997.

[23] I. Queinnec, S. Tarbouriech, and G. Garcia. Anti-windup design for aircraft flight control. IEEE Conference on Control Applications, 2006.

[24] A. Rantzer. A performance criterion for anti-windup compensators. European Journal of Control, 6(5), 2000.

[25] A.A. Rodriguez and J. R. Cloutier. Control of a bank-to-turn missile with saturating actuators. Proc. American Control Conference, 1994.

[26] B.G. Romanchuk. Some comments on anti-windup synthesis using LMI's. International Journal of Robust and Nonlinear Control, 9(10):717-734, 1999.

[27] L. Rundquist and K. Stahl-Gunnarsson. Phase compensation of rate-limiters in unstable aircraft. Proc. IEEE Conference on Control Applications, 1996.

[28] A. Saberi, Z. Lin, and A.R. Teel. Control of linear systems with saturating actuators. IEEE Trans. on Automatic Control, 41(3):368-378, 1996.

[29] A. Saberi and A. Stoorvogel. Output regulation of linear plants with actuators subject to amplitude and rate constraints. Int. J. Robust and Nonlinear Control, 9(10):631-657, 1999.

[30] M. Saeki and N. Wada. Synthesis of a static anti-windup compensator via linear matrix inequalities. International Journal of Robust and Nonlinear Control, 12:927-953, 2002.

[31] J. Sofrony, M.C. Turner, and I. Postlethwaite. Anti-windup synthesis for systems with rate-limits: a Riccati equation approach. Proc. SICE Annual Conference, 2005.

[32] J. Sofrony, M.C. Turner, and I. Postlethwaite. Anti-windup synthesis using Riccati equations. Proc. IFAC World Congress, 2005.

[33] J. Sofrony, M.C. Turner, and I. Postlethwaite. Anti-windup synthesis using Riccati equations. International Journal of Control, 80(1):112-128, 2007.

[34] J. Sofrony, M.C. Turner, I. Postlethwaite, O.M. Brieger, and D. Leissling. Anti-windup synthesis for PIO aviodance in an experimental aircraft. IEEE Conference on Decision and Control, 2006.

[35] G. Stein. Respect the unstable. IEEE Control Systems Magazine, pages 12-25, 2003.

[36] R. Suarez, J. Alvaerz-Ramirez, and J. Solis-Daun. Linear systems with bounded inputs: global stabilisation with eigenvalue placement. International Journal of Robust and Nonlinear Control, 7:835-845, 1997.

[37] S. Tarbouriech, G. Garcia, and A.H. Glattfelder. Advanced Strategies in Control Systems with input and Output Constraints. Springer-Verlag, London, 2006. 
[38] S. Tarbouriech, I. Queinnec, and G. Garcia. Stability region enlargement through anti-windup strategy for linear systems with dynamics restricted actuator. International Journal of Systems Science, 37(2):79-90, 2006.

[39] A.R. Teel. Anti-windup for exponentially unstable linear systems. international Journal of Robust Nonlinear Control, pages 701-716, 1999.

[40] A.R. Teel and N. Kapoor. The $\mathcal{L}_{2}$ anti-windup problem: Its definition and solution. Proc. European Cont. Conf., 1997.

[41] M.C. Turner and I. Postlethwaite. Guaranteed stability regions of linear systems subject to actuator saturation using the low-and-high gain technique. International Journal of Control, 74(14):14251434, 2001.

[42] M.C. Turner and I. Postlethwaite. A new perspective on static and low order anti-windup synthesis. International Journal of Control, 77(1):27-44, 2004.

[43] P.F. Weston and I Postlethwaite. Analysis and design of linear conditioning schemes for systems containing saturating actuators. IFAC Nonlinear Control System Design Symposium, 1998.

[44] P.F. Weston and I Postlethwaite. Linear conditioning for systems containing saturating actuators. Automatica, 36(9):1347-1354, 2000.

[45] F. Wu and M. Soto. Extended anti-windup control schemes for LTI and LFT systems with actuator saturations. International Journal of Robust and Nonlinear Control, 14(15):1255-1281, 2004. 